\title{
Lack of association between genetic variants in the 19q13.32 region and CHD risk in the Algerian population: a population-based nested case-control study
}

\author{
Houssam Boulenouar ${ }^{1,2,3}$, Sarah Aicha Lardjam Hetraf ${ }^{3}$, Hadjira Ouhaibi Djellouli ${ }^{3}$, Djabaria Naima \\ Meroufel $^{3}$, Faouzia Zemani Fodil ${ }^{3}$, Imane Hammani-Medjaoui ${ }^{4}$, Nadhira Saidi Mehtar ${ }^{3}$, \\ Leila Houti ${ }^{5}$, Sounnia Mediene Benchekor ${ }^{3,6}$
}

1. Laboratoire de recherche Cancer Lab N³0, Faculté de Médecine « Dr Benzerdjeb Benaouda », Université Aboubekr Belkaid- Tlemcen 13000, Algérie.

2. Département de Médecine, Faculté de Médecine «Dr Benzerdjeb Benaouda », Université Aboubekr Belkaid- Tlemcen 13000, Algérie.

3. Laboratoire de Génétique Moléculaire et Cellulaire, Université des sciences et de la technologie d'OranMohamed BOUDIAF, Oran 31000, Algérie.

4. Caisse Nationale des Assurances Sociales des travailleurs salariés, Clinique Spécialisée en Orthopédie et Rééducation des Victimes des Accidents de Travail, Oran 31000, Algérie.

5. Faculté de Médecine, Université d'Oran 1 and LABoratoire des Systèmes d'Information en Santé, Université d'Oran 1, Oran 31000, Algérie.

6. Département de Biotechnologie, Faculté des Sciences de la Nature et de la Vie, Université Oran 1 Ahmed Ben Bella, Oran 31000, Algérie.

\begin{abstract}
Background: Coronary Heart Disease (CHD) is a major cause of morbidity and mortality over the world; intermediate traits associated with CHD commonly studied can be influenced by a combination of genetic and environmental factors. Objective: We found previously significant association between three genetic polymorphisms, and the lipid profile variations in the Algerian population. Considering these findings, we therefore decided to assess the relationships between these polymorphisms and CHD risk,

Methods: We performed a population-based, cross-sectional study, of 787 individuals recruited in the city of Oran, in which, a nested case-control study for MetS, T2D, HBP, obesity and CHD were performed. Subjects were genotyped for four SNP rs7412, rs429358 rs4420638 and rs439401 located in the 19q13.32 region.

Results: The T allele of rs439401 confers a high risk of hypertension with an odds ratio (OR) of 1.46 (95\% CI [1.12-1.9], p $=0.006)$ and the $\mathrm{G}$ allele of rs 4420638 was significantly associated with a decreased risk of obesity, OR 0.48 (95\% CI [0.290.81], $\mathrm{p}=0.004)$. No associations were found for MetS, T2D and CHD.

Conclusion: Although the studied genetic variants were not associated with the risk of CHD, the 19q13.32 locus was associated with some of the cardiometabolic disorders in Algerian subjects.

Keywords: Genetics; high blood pressure; CHD risk; obesity; Algerian population.

DOI: https://doi.org/10.4314/ahs.v20i2.25

Cite as: Boulenouar H, Hetraf SAL, Djellouli HO, Meroufel DN, Fodil FZ, Hammani-Medjaoni I, et al. Lack of association between genetic variants in the $19 q 13.32$ region and CHD risk in the Algerian population: a population-based nested case-control study. Afri Health Sci. 2020; 20(2): 735-744. https://doi.org/10.4314/abs.v20i2.25
\end{abstract}

\section{Corresponding author:}

Houssam Boulenouar,

Département de Médecine, Faculté de Médecine

«Dr Benzerdjeb Benaouda », Université Aboubekr

Belkaid- Tlemcen 13000, Algérie.

Tel : +213771447897 / +213550376034

Email: boulenouar.houssam@gmail.com/

houssam.boulenouar@mail.univ-tlemcen.dz

\section{Background and aims}

Coronary Heart Disease (CHD) is a major cause of morbidity and mortality over the world; indeed, deaths from ischemic heart disease in 2008 were estimated at 7.3 millions in the world (12.4\% of all mortality) and predictions for the year 2020 suggest that CHD will remain the first cause of death ${ }^{2}$.

Intermediate traits associated with CHD commonly studied include body mass index (BMI), blood pres-

(C) 2020 Boulenouar H et al. Licensee African Health Sciences. This is an Open Access article distributed under the terms of the Creative commons Attribution License (https://creativecommons.org/licenses/BY/4.0), which permits unrestricted use, distribution, and reproduction in any medium, provided the original work is properly cited. 
sure and cholesterol plasma levels (total cholesterol, LDL-cholesterol and HDL-cholesterol), which can be influenced by a combination of genetic ${ }^{3}$ and environmental factors (such as diet, alcohol, and physical ac-

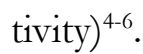

Several meta-analyses indicated that the APOE epsilon polymorphism is a genetic risk factor for $\mathrm{CHD}^{7-10}$. Furthermore, the APOE gene located on 19q13.32 locus was reported to be significantly associated with plasma cholesterol concentration variations; The APOE $\varepsilon 4$ allele is associated with higher total and LDL-cholesterol levels and a higher risk of CHD, whereas the APOE $\varepsilon 2$ allele presents the opposite protective effects in Caucasian populations ${ }^{11-14}$.

Two other polymorphisms situated in the $19 \mathrm{q} 13.32$ locus showing associations with various metabolic traits have been studied. The rs439401 SNP was associated with higher plasma triglyceride and lower plasma HDL-cholesterol concentrations ${ }^{10,15}$ and the rs4420638 SNP which was associated with lower plasma HDL-cholesterol levels, higher total cholesterol and LDL-cholesterol concentrations, higher total-cholesterol/HDL-cholesterol and LDL-cholesterol/HDL-cholesterol ratios ${ }^{10,16-18}$.

In our previous study, we found significant association between the three studied polymorphisms (Epsilon, rs439401 and rs4420638) located in the $19 q 13.32$ region, and the lipid profile variations in the Algerian population (1). Considering these findings, we therefore decided firstly to assess the relationships between APOE epsilon, rs439401 and rs4420638 polymorphisms and CHD risk, then thereafter, we aimed to analyze the association of the three polymorphisms with Metabolic syndrome (MetS), obesity, Type 2 diabètes (T2D) and high blood pressure (HBP) risk, considered as established risk factors for $\mathrm{CHD}$, in a general population sample from the city of Oran in north-west Algeria, the ISOR study, by performing a nested case-control study. To the best of our knowledge, the relationship between the studied polymorphisms and CHD, MetS, T2D, obesity and HBP risk has never previously been studied in an Algerian population.

\section{Material and methods}

\section{Ethical considerations}

The work has been done according to Helsinki Declaration and the study's objectives and procedures were approved by the independent ethics committee at the Algerian National Agency for the Development of Health Research (ANDRS), (since renamed as the Thematic Agency of Research in Health Sciences ATRSS).
All participants provided written informed consent prior to enrolment.

\section{Study population}

Participants were recruited during the ISOR (InSulino-résistance à ORan) study, a population-based, cross-sectional study of a representative sample of 787 individuals (378 men and 409 women, aged between 30 and 64 years) recruited within the city of Oran, Algeria, from 2007 to 2009, Subjects were selected at random from social security rollsdetails of the ISOR study have been described elsewhere ${ }^{1}$.

\section{Data collection}

Data were collected using a pretested questionnaire on socioeconomic information, physical activity (The level of physical activity was defined in quartiles as "none", "low", "medium" and "high" after summing exercise scores for sporting activities, walking, housework and physical activity at work), tobacco use and alcohol intake, past medical history and family history, current medications, as well as anthropomorphic characteristics including height, weight, waist circumference, hip circumference, and blood pressure. Height and weight were measured while the subject was barefoot and lightly dressed. The BMI was calculated according to the Quetelet equation. Systolic and diastolic blood pressure values (SBP and DBP, respectively) were measured on the right arm with the subject in the sitting position, using a standard mercury sphygmomanometer. Measurements were made before and after completion of the questionnaire, with an interval of at least $10 \mathrm{~min}-$ utes. The mean value of the blood pressure readings was considered for analysis. Regarding tobacco use, participants were categorized as either smokers (i.e. individuals reporting at least one cigarette per day) or non-smokers. After a 12 hour overnight fast, blood was collected aseptically via venipuncture in an EDTA tube for DNA extraction and subsequent molecular analysis, and in a heparin tube for biochemistry tests.

\section{CHD diagnosis criteria}

Subjects who self reported previously having at least one of the diseases among angina pectoris, myocardial infarction, stroke or peripheral artery disease, were considered as CHD cases.

\section{Cardiometabolic risk factors diagnosis criteria}

In this study, we have adopted the definition of metabolic syndrome according to the criteria of the NCEP ATPIII (National Cholesterol Education Program- 
Adult Treatment Panel III) ${ }^{19}$, the metabolic syndrome is diagnosed when a subject has three or more of the following risk factors:

- Abdominal obesity: waist circumference $>102 / 88$ cm (Men/Women);

- Triglyceride level $\geq 1,50 \mathrm{~g} / \mathrm{l}(1,69 \mathrm{mmol} / \mathrm{l})$;

- HDL-cholestérol <0.40/0.50 g/l (1.04/1.29 mmol/l)

(Men/Women);

- Blood pressure $\geq 130 / 85 \mathrm{mmHg}$;

- Fasting glucose $\geq 100 \mathrm{mg} / \mathrm{dl}(5,6 \mathrm{mmol} / \mathrm{l})$.

For Type 2 diabetes, the definition adopted in this study is that of the American Diabetes Association (ADA) ${ }^{20}$.

- Fasting plasma glucose $\geq 1.26 \mathrm{~g} / 1(7 \mathrm{mmol} / \mathrm{l})$ twice after 8 hours of fasting

- Occasional blood glucose $\geq 2 \mathrm{~g} / 1(11.1 \mathrm{mmol} / \mathrm{l})$ in the presence of symptoms of hyperglycemia (polyuria, polydipsia, unexplained weight loss)

- Diabetics declared under treatment including oral antidiabetic and/or insulin

Hypertension (HBP) has been defined according to WHO criteria ${ }^{21}$ : mean systolic blood pressure greater than $140 \mathrm{~mm} \mathrm{Hg}$ and/or mean diastolic blood pressure greater than $90 \mathrm{mmHg}$ and/or self-reported current treatment for hypertension with antihypertensive drugs. The body mass index (BMI) is calculated according to the Quetelet equation. A subject is considered obese if he has a BMI greater than or equal to $30 \mathrm{~kg} / \mathrm{m}^{2}$.

\section{Biochemistry testing}

A multichannel analyzer and dedicated kits (Humas$\operatorname{tar}{ }^{\circledR}$, HUMAN Diagnostics, Wiesbaden, Germany) were used for the colorimetric, enzymatic measurement of cholesterol (kit: monotest cholesterol with cholesterol esterase, cholesterol oxidase and peroxidase), triglycerides (kit: peridochrom triglyceride with glycerol phosphate oxidase and peroxidase) and glucose (kit: glucose, glucose oxidase and peroxidase). Plasma LDL-cholesterol levels were calculated according to the Friedewald equation. High-density lipoprotein cholesterol levels were measured after sodium phosphotungstate/magnesium chloride precipitation of chylomicrons and VLDL and LDL-cholesterol and then centrifugation. Plasma insulin levels were measured using a microparticle enzyme immune assay running on an AxSYM analyzer (Abbott Laboratories, Abbott Park, Illinois, USA).

\section{Genotyping}

Genomic DNA was extracted from white blood cells by using the Stratagene ${ }^{\circledR}$ kit (Agilent Technologies, Les
Ulis, France), according to the manufacturer's protocol. Three genetic polymorphisms were characterized in this study, the Epsilon polymorphism defined by defined by the rs7412 and rs429358 single nucleotide polymorphisms (SNPs), the rs439401 and the rs4420638, genotyping was performed by using KASPar technology (KBioscience, Hoddesdon, UK), a competitive allele-specific polymerase chain reaction incorporating a fluorescent resonance energy transfer quencher cassette (for more information see http://www.kbioscience.co.uk/reagents/KASP.html). The KASPar assay was designed using the Primer Picker software available at http://www.kbioscience.co.uk/primer-picker.htm (KBioscience). Genotyping assays was carried outwith a Hydrocycler (Applied Biosystems, Foster City, CA) in a final volume of $2 \mu \mathrm{l}$ containing $4 \times$ Reaction Mix (KBioscience), $120 \mathrm{nM}$ of each allele-specific primer and $300 \mathrm{nM}$ of common primer, $1.5 \mu \mathrm{l}$ of Master mix (KBioscience) and $5 \mathrm{ng}$ of genomic DNA. Appropriate negative control samples were used.

The genotyping success rates ranged from $93 \%$ to $96 \%$. Around $9 \%$ of the participants of the ISOR study was double genotyped, with a $100 \%$ concordance rate.

\section{Statistical analysis}

Statistical analyses were performed with SAS 9.1 software (SAS Institute Inc., Cary, NC, USA). The Hardy-Weinberg equilibrium was tested using a $\chi^{2}$ test with one degree of freedom (d.f.). Some of the biochemical traits (Fasting Glucose levels, Triglycerides and Insulin levels) were not normally distributed, we therefore log-transformed these parameters to obtain normal data distributions. Intergroup comparisons of means were performed with a general linear model, multivariate logistic regression analyses were used to calculate the odds ratios for CHD, MetS, T2D, HBP and obesity. The confounding variables were age, gender, smoking status and physical activity. After Bonferroni correction, only associations with an uncorrected $\mathrm{p}$ value below 0.017 were considered to be statistically significant.

The statistical power was estimated using QUANTO software (http://hydra.usc.edu/gxe/).

\section{Results \\ Characteristics of study subjects}

The main anthropometric, biochemical and clinical characteristics have been measured; the baseline characteristics of the ISOR population study were summarized in Table 1 
Table 1: Baseline characteristics of the study population

\begin{tabular}{|c|c|c|c|c|c|c|c|}
\hline \multirow[b]{2}{*}{ Parameters } & \multicolumn{2}{|c|}{ All $(\mathrm{n}=787)$} & \multicolumn{2}{|c|}{ Non $\mathrm{CHD}(\mathrm{n}=665)$} & \multicolumn{2}{|c|}{$\mathrm{CHD}(\mathrm{n}=122)$} & \multirow[b]{2}{*}{$p$} \\
\hline & Mean & SD & Mean & SD & Mean & $\mathrm{SD}$ & \\
\hline Age (yrs) & 44.0 & 10.1 & 43,7 & 10,2 & 45.6 & 9.8 & $0.007 *$ \\
\hline Height (cm) & 165.5 & 9.6 & 165.4 & 9,5 & 165,9 & 10,3 & ns \\
\hline Weight (kg) & 71.3 & 14.6 & 70,9 & 14.9 & 73,3 & 13,3 & $5 \times 10^{s *}$ \\
\hline $\operatorname{BMI}\left(\mathrm{kg} / \mathrm{m}^{2}\right)$ & 26.1 & 5.1 & 26 & 5,2 & 26,7 & 4,6 & $0,04 *$ \\
\hline Waist (cm) & 87.7 & 12.4 & 87,2 & 12,3 & 91,2 & 13,4 & $2.2 \times 10^{3 *}$ \\
\hline Waist-to-hip ratio & 0.86 & 0.09 & 0,86 & 0,08 & 0,88 & 0,1 & $0,006^{*}$ \\
\hline Fasting plasma glucose (mmol/L) & 5.34 & 1.85 & 5.20 & 1.84 & 5.45 & 1.85 & $0.001^{*}$ \\
\hline Fasting insulin $(\mu \mathrm{UI} / \mathrm{mL})$ & 8.44 & 6.54 & 8,38 & 6,61 & 8,75 & 6,12 & $2.2 \times 10^{-14 *}$ \\
\hline Total Cholesterol(mmol/L) & 4.45 & 0.91 & 4.45 & 0.76 & 4.44 & 1.03 & ns \\
\hline Triglycerides $(\mathrm{mmol} / \mathrm{L})$ & 1.17 & 0.51 & 1.02 & 0,45 & 1,12 & 0,45 & $0.04 *$ \\
\hline HDL- cholesterol(mmol/L) & 1.25 & 0.31 & 1.27 & 0.30 & 1.23 & 0.32 & ns \\
\hline LDL- cholesterol(mmol/L) & 2.68 & 0.87 & 2.64 & 0.73 & 2.72 & 1.10 & ns \\
\hline SBP (mm Hg) & 123.6 & 18.1 & 123 & 17,6 & 126,5 & 19,7 & $0.01 *$ \\
\hline $\mathrm{DBP}(\mathrm{mm} \mathrm{Hg})$ & 76.9 & 9.9 & 76,5 & 9,7 & 75,9 & 12,9 & $0.02 *$ \\
\hline
\end{tabular}

Prevalence of the metabolic syndrome and the sented in Table 2. main cardiometabolic risk factors

These data concerning the Oran population are pre-

Table 2: Prevalence of cardiometabolic disorders among men and women in the ISOR population

\begin{tabular}{|c|c|c|c|c|}
\hline & $\frac{\text { All, } n=787}{\mathrm{~N}(\%)}$ & $\frac{\text { Men, } n=378}{\mathrm{~N}(\%)}$ & $\frac{\text { Women, } n=409}{\mathrm{~N}(\%)}$ & $p$ \\
\hline Abdominal Adiposity & $233(30.1)$ & $45(12.1)$ & $188(46.8)$ & $<0001 *$ \\
\hline High Triglycerides & $103(13.3)$ & $62(16.7)$ & $41(10.2)$ & 0.63 \\
\hline Low HDL-Cholesterol & $342(44.2)$ & 99 (26.6) & $243(60.4)$ & $<0001 *$ \\
\hline High Fasting Glucose & $161(20.8)$ & 88 (23.7) & $73(18.2)$ & 0,47 \\
\hline $\mathrm{T} 2 \mathrm{D}$ & $80(10.6)$ & 42 (11.6) & $38(9.7)$ & 0.39 \\
\hline Obesity & $167(21.2)$ & $34(9.0)$ & $133(32.5)$ & $<0001 *$ \\
\hline MetS & $155(20.0)$ & $51(13.7)$ & $104(25.9)$ & $<0001 *$ \\
\hline HBP & $160(20.3)$ & $80(21.2)$ & $80(19.6)$ & 0.58 \\
\hline CHD & $122(15.5)$ & $59(15.6)$ & $63(15.4)$ & 0.96 \\
\hline
\end{tabular}

T2D: Type 2 Diabetes, MetS : Metabolic Syndrome, HBP : High Blood Pressure, CHD : Coronary heart disease,

$\mathrm{N}$ : Number, \%: Percentage, $p$ values obtained for intergroup comparison chi-square test

Significant $p$ values are indicated in bold with an asterisk

Diabetes mellitus (T2D) was diagnosed in 80 participants $(10.6 \%)$. The distribution of prevalence by sex shows no significant difference $(p=0.39)$, it was $11.6 \%$ for men and $9.7 \%$ for women.

The prevalence of obesity in the general population is $21.2 \%$. It affects more women $(32.5 \%)$ than men $(9 \%)$, with a significant difference in the prevalence distribution between men and women ( $\mathrm{p}<0.0001)$.

Abdominal adiposity were present in 233 subjects, it represents 301 of the total population, with a feminine predominance $(46.8 \%)$ in women vs $(12.1 \%)$ in men $(\mathrm{p}<0.0001)$

High triglyceride and high fasting glucose levels were present in $13.3 \%$ and $20.8 \%$ of the total population respectively, there no significant difference among men and women.

The prevalence of the metabolic syndrome in the Oran population is $20 \%$, the distribution of this pathology is also significantly different between the two sexes ( $p$ $<0.0001)$. Indeed, it affects more women $(25.9 \%)$ than men $(13.7 \%)$. 
Hypertension affects $23.1 \%$ of the study population. HBP is present in $21.2 \%$ of men and $19.6 \%$ of women, the prevalence distribution in men and women shows no significant difference $(p=0.57)$.

One hundred twenty two subjects corresponding to $15.5 \%$ of the total population presented a CHD; there was no significant difference in distribution between men and women.

\section{Genotype and allele distributions}

We have determined the allele and genotype distributions of three polymorphisms (Epsilon, rs439401 and rs4420638) in order to evaluate their possible association with the risk of CHD, MetS, T2D, HBP and Obes; results were described in Table 3. There was no evidence of significant deviation from Hardy-Weinberg equilibrium in any distributions.

Table 3: Genotype and allele distribution among subjects with CHD, MetS, T2D, HBP, Obes and controls

\begin{tabular}{|c|c|c|c|c|c|c|c|c|c|c|c|}
\hline \multirow{10}{*}{ Epsilon } & \multirow{3}{*}{$\begin{array}{c}\text { Genotype } \\
\text { Frequency } \\
\varepsilon 2 / \varepsilon 2 \\
\varepsilon 2 / \varepsilon 3\end{array}$} & \multirow{2}{*}{$\begin{array}{l}\text { CHD } \\
\mathrm{N}(\%)\end{array}$} & \multirow{2}{*}{$\frac{\text { Non CHD }}{\mathrm{N}(\%)}$} & \multirow{2}{*}{$\begin{array}{l}\text { MetS } \\
\mathrm{N}(\%)\end{array}$} & \multirow{2}{*}{$\frac{\text { Non MetS }}{\mathrm{N}(\%)}$} & \multirow{2}{*}{$\begin{array}{c}\text { T2D } \\
\mathrm{N}(\%)\end{array}$} & \multirow{2}{*}{$\frac{\text { Non T2D }}{\mathrm{N}(\%)}$} & \multirow{2}{*}{$\begin{array}{l}\text { HBP } \\
\mathrm{N}(\%)\end{array}$} & \multirow{2}{*}{$\frac{\text { Non HBP }}{\mathrm{N}(\%)}$} & \multirow{2}{*}{$\begin{array}{l}\text { Obes } \\
\mathrm{N}(\%)\end{array}$} & \multirow{2}{*}{$\frac{\text { Non Obes }}{\mathrm{N}(\%)}$} \\
\hline & & & & & & & & & & & \\
\hline & & $\begin{array}{c}0 \\
10(8.7)\end{array}$ & $\begin{array}{c}7(1.1) \\
51(8.2)\end{array}$ & $\begin{array}{c}1(0.7) \\
14(9.7)\end{array}$ & $\begin{array}{c}5(0.9) \\
43(7.4)\end{array}$ & $\begin{array}{c}0 \\
5(6.7)\end{array}$ & $\begin{array}{c}7(1.1) \\
49(7.8)\end{array}$ & $\begin{array}{c}1(0.7) \\
11(7.4)\end{array}$ & $\begin{array}{c}6(1.0) \\
49(8.4)\end{array}$ & $\begin{array}{c}2(1.3) \\
19(12.6)\end{array}$ & $\begin{array}{c}5(0.8) \\
42(7.2)\end{array}$ \\
\hline & $\begin{array}{l}\varepsilon 2 / \varepsilon 4 \\
\varepsilon 3 / \varepsilon 3 \\
\varepsilon 3 / \varepsilon 4\end{array}$ & $\begin{array}{c}0 \\
89(77.4) \\
14(12.2)\end{array}$ & $\begin{array}{c}3(0.5) \\
483(77.5) \\
71(11.4)\end{array}$ & $\begin{array}{c}0 \\
109(75.7) \\
16(11.1)\end{array}$ & $\begin{array}{c}3(0.5) \\
457(78.8) \\
66(11.4)\end{array}$ & $\begin{array}{c}0 \\
63(84) \\
7(9.3)\end{array}$ & $\begin{array}{c}3(0.5) \\
491(77.8) \\
71(11.2)\end{array}$ & $\begin{array}{c}0 \\
118(80.3) \\
15(10.2)\end{array}$ & $\begin{array}{c}3(0.5) \\
450(76.8) \\
70(11.9)\end{array}$ & $\begin{array}{c}0 \\
115(76.2) \\
13(8.6)\end{array}$ & $\begin{array}{c}3(0.5) \\
456(77.8) \\
72(12.3)\end{array}$ \\
\hline & $\begin{array}{c}\varepsilon 4 / \varepsilon 4 \\
\text { TOTAL }\end{array}$ & $\begin{array}{c}2(1.7) \\
115(100)\end{array}$ & $\begin{array}{c}8(1.3) \\
623(100)\end{array}$ & $\begin{array}{c}4(2.8) \\
144(100)\end{array}$ & $\begin{array}{c}6(1) \\
580(100)\end{array}$ & $\begin{array}{c}0 \\
75(100)\end{array}$ & $\begin{array}{c}10(1.6) \\
631(100)\end{array}$ & $\begin{array}{c}2(1.4) \\
147(100)\end{array}$ & $\begin{array}{c}8(1.4) \\
586(100)\end{array}$ & $\begin{array}{c}2(1.3) \\
151(100)\end{array}$ & $\begin{array}{c}8(1.4) \\
586(100)\end{array}$ \\
\hline & $p$ & \multicolumn{2}{|c|}{0.83} & \multicolumn{2}{|c|}{0.51} & \multicolumn{2}{|c|}{0.69} & \multicolumn{2}{|c|}{0.91} & \multicolumn{2}{|c|}{0.25} \\
\hline & $\begin{array}{c}\text { Allele } \\
\text { frequency }\end{array}$ & $(\%)$ & $(\%)$ & $(\%)$ & $(\%)$ & $(\%)$ & $(\%)$ & $(\%)$ & $(\%)$ & $(\%)$ & $(\%)$ \\
\hline & $\varepsilon 2$ & 4.3 & 5.4 & 5.5 & 4.8 & 3.3 & 5.2 & 4.4 & 5.5 & 7.6 & 4.7 \\
\hline & 83 & 87.9 & 87.4 & 86.1 & 88.2 & 92.0 & 87.3 & 89.1 & 86.9 & 86.8 & 87.5 \\
\hline & $\varepsilon 4$ & 7.8 & 7.2 & 8.4 & 7 & 4.7 & 7.5 & 6.5 & 7.6 & 5.6 & 7.8 \\
\hline \multirow[t]{9}{*}{$\begin{array}{c}\text { rs } 43940 \\
1\end{array}$} & $\begin{array}{l}\text { Genotype } \\
\text { Frequency }\end{array}$ & $\mathrm{N}(\%)$ & $\mathrm{N}(\%)$ & $\mathrm{N}(\%)$ & $\mathrm{N}(\%)$ & $\mathrm{N}(\%)$ & $\mathrm{N}(\%)$ & $\mathrm{N}(\%)$ & $\mathrm{N}(\%)$ & $\mathrm{N}(\%)$ & $\mathrm{N}(\%)$ \\
\hline & $\mathrm{CC}$ & $42(36.8)$ & $248(39.5)$ & $56(38.6)$ & $228(39.1)$ & $22(28.9)$ & $254(40.1)$ & $49(33.3)$ & $241(40.9)$ & $57(36.3)$ & $233(39.9)$ \\
\hline & CT & $57(50.0)$ & $280(44.6)$ & $65(44.8)$ & $265(45.2)$ & $38(50.0)$ & $285(45.0)$ & $74(50.3)$ & $262(44.5)$ & $74(47.1)$ & $262(44.9)$ \\
\hline & TT & $15(13.2)$ & $100(15.9)$ & $24(16.6)$ & $90(15.4)$ & $16(21.1)$ & $95(15.0)$ & $24(16.3)$ & $86(14.6)$ & $26(16.6)$ & $89(15.2)$ \\
\hline & TOTAL & $114(100)$ & $628(100)$ & $145(100)$ & $583(100)$ & $76(100)$ & $634(100)$ & $147(100)$ & $589(100)$ & $157(100)$ & $584(100)$ \\
\hline & $p$ & \multicolumn{2}{|c|}{0.52} & \multicolumn{2}{|c|}{0.94} & \multicolumn{2}{|c|}{0.12} & \multicolumn{2}{|c|}{0.24} & \multicolumn{2}{|c|}{0.70} \\
\hline & $\begin{array}{c}\text { Allele } \\
\text { frequency }\end{array}$ & $(\%)$ & $(\%)$ & $(\%)$ & $(\%)$ & $(\%)$ & $(\%)$ & $(\%)$ & $(\%)$ & $(\%)$ & $(\%)$ \\
\hline & $\mathrm{C}$ & 61.8 & 61.8 & 61 & 61.8 & 53.9 & 62.5 & 58.5 & 63.2 & 59.9 & 62.3 \\
\hline & $\mathrm{T}$ & 38.2 & 38.2 & 39 & 38.2 & 46.1 & 37.5 & 41.5 & 36.8 & 40.1 & 37.7 \\
\hline \multirow[t]{9}{*}{$\begin{array}{c}\text { rs } 44206 \\
38\end{array}$} & $\begin{array}{l}\text { Genotype } \\
\text { Frequency }\end{array}$ & $\mathrm{N}(\%)$ & $\mathrm{N}(\%)$ & $\mathrm{N}(\%)$ & $\mathrm{N}(\%)$ & $\mathrm{N}(\%)$ & $\mathrm{N}(\%)$ & $\mathrm{N}(\%)$ & $\mathrm{N}(\%)$ & $\mathrm{N}(\%)$ & $\mathrm{N}(\%)$ \\
\hline & $\mathrm{AA}$ & $91(78.4)$ & $509(79.2)$ & $117(79.6)$ & $473(79.1)$ & $63(79.7)$ & $515(79.5)$ & $127(13.3)$ & $469(77.6)$ & $136(86.6)$ & $463(77.0)$ \\
\hline & AG & $24(20.7)$ & $125(19.4)$ & $27(18.4)$ & $118(19.7)$ & $16(18.3)$ & $124(19.1)$ & $20(84.7)$ & $128(21.2)$ & $21(13.4)$ & $128(21.3)$ \\
\hline & GG & $1(09)$ & $9(1.4)$ & $3(2)$ & $7(1.2)$ & 0 & $9(1.4)$ & $3(2)$ & $7(1.2)$ & 0 & $10(1.7)$ \\
\hline & TOTAL & $116(100)$ & $643(100)$ & $147(100)$ & $598(100)$ & $79(100)$ & $648(100)$ & $150(100)$ & $604(100)$ & $157(100)$ & $601(100)$ \\
\hline & $p$ & \multicolumn{2}{|c|}{0.86} & \multicolumn{2}{|c|}{0.67} & \multicolumn{2}{|c|}{0.56} & \multicolumn{2}{|c|}{0.07} & \multicolumn{2}{|c|}{$0.02^{*}$} \\
\hline & $\begin{array}{l}\text { Allele } \\
\text { frequency }\end{array}$ & $(\%)$ & $(\%)$ & $(\%)$ & $(\%)$ & $(\%)$ & $(\%)$ & $(\%)$ & $(\%)$ & $(\%)$ & $(\%)$ \\
\hline & A & 88.8 & 88.9 & 88.8 & 89 & 89.9 & 89 & 91.3 & 88.2 & 93.3 & 87.7 \\
\hline & G & 11.2 & 11.1 & 11.2 & 11 & 10.1 & 11 & 8.7 & 11.8 & 6.7 & 12.3 \\
\hline
\end{tabular}

N Number, \% Percentage, $p$ values obtained for intergroup comparison chi-square test, Significant $p$ values are indicated in bold with an asterisk 
Table 4: Association of the three studied polymorphisms with cardiometabolic risk factors

\begin{tabular}{|c|c|c|c|c|c|c|c|c|c|c|c|}
\hline & \multicolumn{3}{|c|}{ Epsilon polymorphism } & \multicolumn{4}{|c|}{ rs 439401} & \multicolumn{4}{|c|}{ rs4420638 } \\
\hline & \multirow{2}{*}{\multicolumn{2}{|c|}{$\begin{array}{c}\text { Alleles }(\mathrm{N}) \\
p\end{array}$}} & \multirow{2}{*}{$\begin{array}{c}\text { OR }[95 \% \mathrm{CI}] \\
p \\
\end{array}$} & \multirow{2}{*}{\multicolumn{2}{|c|}{$\begin{array}{c}\text { Genotypes(N) } \\
p \\
\end{array}$}} & \multirow{2}{*}{$\begin{array}{c}\text { OR }[95 \% \mathrm{Cl}] \\
p \\
\end{array}$} & \multirow{2}{*}{$\begin{array}{c}\text { OR: }[95 \% \mathrm{CI}] \\
p \\
\end{array}$} & \multicolumn{2}{|c|}{ Genotypes (N) } & \multirow{2}{*}{$\begin{array}{c}\text { OR }[95 \% \mathrm{Cl}] \\
p \\
\end{array}$} & \multirow{2}{*}{$\begin{array}{c}\text { OR' }[95 \% \mathrm{CI}] \\
p \\
\end{array}$} \\
\hline & & & & & & & & $p$ & $p$ & & \\
\hline \multirow{6}{*}{ T2D } & Control & Case & $\varepsilon 2$ vs $\varepsilon 3$ : & Control & Case & CT vs CC: & & Control & Case & AG vs AA: & \\
\hline & $\varepsilon 2(52)$ & $\varepsilon 2(6)$ & $0,87[0,34-2,22]$ & CC (254) & $\mathrm{CC}(22)$ & $1,57[0,88-2,79]$ & $1,47[1,03-2,09]$ & $\mathrm{AA}(515)$ & AA (63) & $1,20[0,65-2,22]$ & $0,99[0,56-1,75]$ \\
\hline & $\varepsilon 3(455)$ & $\varepsilon 3(58)$ & $p=0,77$ & CT (285) & CT (38) & $p=0,13$ & $p=0,03$ & $\mathrm{AG}(124)$ & $\operatorname{AG}(16)$ & $p=0,56$ & $p=0,98$ \\
\hline & $\varepsilon 4(116)$ & $\varepsilon 4(12)$ & $\varepsilon 4$ vs $\varepsilon 3$ : & TT (95) & TT (16) & TT vs CC : & & GG $(9)$ & GG $(0)$ & & \\
\hline & & & $0,84[0,42-1,68]$ & & & $2,12[1,02-4,39]$ & & & & & \\
\hline & \multicolumn{2}{|c|}{$p=0,81$} & $p=0,63$ & \multicolumn{2}{|c|}{$p=0,13$} & $p=0,04$ & & \multicolumn{2}{|c|}{$p=0,56$} & & \\
\hline \multirow{5}{*}{ obesity } & $\varepsilon 2(47)$ & $\varepsilon 2(20)$ & $\varepsilon 2$ vs $\varepsilon 3:$ & CC (233) & $\mathrm{CC}(57)$ & CA vs CC: & $1,16[0,89-1,51]$ & $\mathrm{AA}(463)$ & $\mathrm{AA}(136)$ & AG vs AA: & \\
\hline & $\varepsilon 3(414)$ & $\varepsilon 3(111)$ & $1,88[1,01-3,51]$ & CT (262) & CT (74) & $1,27(0,84-1,92]$ & $p=0,27$ & $\mathrm{AG}(128)$ & $\operatorname{AG}(21)$ & $0,51[0,30-0,85]$ & $0,48[0,29-0,79]$ \\
\hline & $\varepsilon 4(115)$ & $\varepsilon 4(22)$ & $p=0,04$ & TT (89) & TT (26) & $p=0,26$ & & GG $(10)$ & $\mathrm{GG}(0)$ & $p=0,01$ & $p=0,004$ \\
\hline & & & $\begin{array}{c}\varepsilon 4 \text { vs } \varepsilon 3: \\
0,80[0,48-1,37]\end{array}$ & & & $\begin{array}{l}\text { AA vs CC: } \\
134[0.76-2.35]\end{array}$ & & & & & \\
\hline & $p=$ & & $p=0,41$ & & & $p=0,31$ & & $p=1$ &, 02 & & \\
\hline \multirow{5}{*}{ MetS } & $\varepsilon 2(48)$ & $\varepsilon 2$ (18) & ६2 vs $\varepsilon 3:$ & CC (228) & $\mathrm{CC}(56)$ & AT vs AA: & $1,06[0,80-1,40]$ & AA (473) & $\mathrm{AA}(117)$ & AG vs AA: & \\
\hline & $\varepsilon 3(424)$ & $\varepsilon 3(96)$ & $1,74[0,92-3,32]$ & CT (265) & $\mathrm{CT}(65)$ & $1,04[0,68-1,59]$ & $p=0,69$ & $\mathrm{AG}(118)$ & $\mathrm{AG}(27)$ & $0,93[0,57-1,53]$ & $1,10[0,71-1,70]$ \\
\hline & $\varepsilon 4(108)$ & $\varepsilon 4(28)$ & $p=0,09$ & TT $(90)$ & TT (24) & $p=0,87$ & & GG (7) & GG (3) & $p=0,78$ & $p=0,66$ \\
\hline & & & $\begin{array}{c}\varepsilon 4 \text { vs } \varepsilon 3: \\
1,29[0,78-2,14]\end{array}$ & & & $\begin{array}{c}\text { TT vs AA: } \\
1,13[0,64-2,02]\end{array}$ & & & & $\begin{array}{c}\text { GG vs AA: } \\
3,19[0,73-13,89]\end{array}$ & \\
\hline & $p=1$ & & $p=0,33$ & & & $p=0,67$ & & $p=0$ & 68 & $p=0,12$ & \\
\hline \multirow{5}{*}{ HBP } & $\varepsilon 2(54)$ & $\varepsilon 2(13)$ & $\varepsilon 2$ vs $\varepsilon 3$ : & CC (241) & $\mathrm{CC}(49)$ & CT vs $\mathrm{CC}:$ & $1,46[1,12-1,90]$ & AA (469) & AA (254) & AG vs AA: & \multirow{5}{*}{$\begin{array}{c}0,73[0,47-1,14] \\
p=0,17\end{array}$} \\
\hline & $\varepsilon 3(413)$ & $\varepsilon 3(112)$ & $1,18[0,63-2,20]$ & CT (262) & CT(74) & $1,46[0,95-2,26]$ & $p=0,006$ & $\mathrm{AG}(128)$ & $\operatorname{AG}(285)$ & $0,56[0,33-0,96]$ & \\
\hline & $\varepsilon 4(110)$ & $\varepsilon 4(27)$ & $p=0,60$ & $\operatorname{TT}(86)$ & TT(29) & $p=0,09$ & & GG (7) & GG (95) & $p=0,03$ & \\
\hline & & & $\begin{array}{c}\varepsilon 4 \text { vs } \varepsilon 3: \\
0,97[0,60-1,59]\end{array}$ & & & $\begin{array}{c}\text { TT vs CC: } \\
1,90[1,07-3,36]\end{array}$ & & & & $\begin{array}{c}\mathrm{GG} \text { vs AA: } \\
2,24[0,51-9,84]\end{array}$ & \\
\hline & \multicolumn{2}{|c|}{$p=0,87$} & $p=0,92$ & $p=$ & & $p=0,03$ & & \multicolumn{2}{|c|}{$p=0.09$} & $p=0,29$ & \\
\hline
\end{tabular}

N Number, OR Odds ratio, CI Confidence Interval, T2D: Type 2 Diabetes, MetS : Metabolic Syndrome, HBP : High Blood Pressure, Significant $p$ values are indicated in bold with an asterisk

$O R$ : The odds ratios (OR) $(95 \%$ confidence interval $(\mathrm{CI}))$ and the p values were obtained from multivariate logistic regression adjusted on age, sexe, physical activity, smoking status, $O R^{*}$ : The odds ratios (OR) ( $95 \%$ confidence interval (CI) and the p values were obtained from logistic regression analyses using additive adjusted on age, sexe, physical activity, smoking status

The rs4420638 genotype distribution were significantly different among subjects with HBP and controls

\section{Association study of the three polymorphisms with cardiometabolic risk factors}

No significant association was reported between genotypes of APOE epsilon polymorphism and the studied cardiovascular risk factors, (T2D, Obesity, HBP and MetS status), the p values ranged from 0.04 to 0.92 (Table 4).

In the ISOR study, rs439401 showed a significant association with hypertension (HBP). The $\mathrm{T}$ allele confers a high risk of hypertension with an odds ratio (OR) of 1.46 (95\% CI [1.12-1.9], $\mathrm{p}=0.006)$. No associations with T2D, obesity and MetS were detected in the ISOR study (Table 4).
Logistic regression analysis showed that the rs4420638 polymorphism was significantly associated with obesity in the general population. The $G$ allele provides protection against obesity, the resulting $\mathrm{OR}$ is 0.48 (95\% CI [0.29-0.81], $\mathrm{p}=0.004$ ) (Table 4). No effects of rs4420638 polymorphism on T2D, MetS, and HBP were detected in the ISOR study.

The associations described for rs439401 and rs4420638 remained significant even after adjusting for the APOE epsilon polymorphism.

\section{Association study of the three polymorphisms with CHD risk}

The three studied polymorphisms showed no significant associations with CHD risk; obtained $\mathrm{p}$ values ranged from 0.24 to 0.80 , results were summarized in table 5 
Table 5: Association of the three studied polymorphisms with CHD risk

\begin{tabular}{|c|c|c|c|c|c|}
\hline & Allele & $\begin{array}{c}\text { Controls } \\
\mathrm{N}(\%)\end{array}$ & $\begin{array}{c}\text { CHD } \\
\text { sujects } \\
\mathrm{N}(\%)\end{array}$ & OR $[95 \%$ CI] & $p$ value \\
\hline Epsilon & $\begin{array}{l}\varepsilon 2 \\
\varepsilon 3 \\
\varepsilon 4\end{array}$ & $\begin{array}{c}58(4.3) \\
483(87.9) \\
79(7.8)\end{array}$ & $\begin{array}{c}10(5.4) \\
89(87.4) \\
16(7.2)\end{array}$ & $\begin{array}{l}0,91[0,44- \\
1,88] \\
1^{\mathrm{a}} \\
1.11[0,59- \\
2.06]\end{array}$ & $\begin{array}{l}p=0,80 \\
p=0,75\end{array}$ \\
\hline rs439401 & $\begin{array}{l}\mathrm{CC} \\
\mathrm{CT} \\
\mathrm{TT}\end{array}$ & $\begin{array}{l}42(36.8) \\
57(50.0) \\
15(13.2)\end{array}$ & $\begin{array}{l}248(39.5) \\
280(44.6) \\
100(15.9)\end{array}$ & $\begin{array}{l}0,82[0,52- \\
1,29] \\
1^{a} \\
0,68[0,36- \\
1,30]\end{array}$ & $\begin{array}{l}p=0,40 \\
p=0,24\end{array}$ \\
\hline rs4420638 & $\begin{array}{l}\text { AA } \\
\text { AG } \\
\text { GG }\end{array}$ & $\begin{array}{c}91(78.4) \\
24(20.7) \\
1(09)\end{array}$ & $\begin{array}{c}509(79.2) \\
125(19.4) \\
9(1.4)\end{array}$ & $\begin{array}{l}0,92[0,54- \\
1,58] \\
1^{a} \\
0,65[0,08- \\
5,51]\end{array}$ & $p=0,69$ \\
\hline
\end{tabular}

N Number, \% Percentage, OR Odds ratio, CI Confidence Interval, ${ }^{a}$ saved as reference category, OR: The odds ratios (OR) (95\% confidence interval (CI)) and the p values were

obtained from multivariate logistic regression adjusted on age, sexe, physical activity, smoking status

\section{Discussion}

In the present work, we conducted a case-control study consisting of 122 confirmed CHD cases and 665 controls to investigate the relationship between the APOE Epsilon, rs439401 and rs4420638 polymorphisms, located on the 19q13.32 region and the risk of CHD in a Western Algerian population. To the best of our knowledge, this is the first study that evaluates the association of the three genetic variants (epsilon, rs439401 and rs4420638) located on the $19 \mathrm{q} 13.32$ region, with the risk of CHD and the main cardiometabolic risk factors, within the Algerian population.

We found no association between the three studied polymorphisms and the metabolic syndrome in the Algerian population. However, some of metabolic disorders considered as cardiometabolic risk factors were significantly associated with the studied polymorphisms.

The logistic regression results showed no association between APOE Epsilon polymorphism and the risk of obesity in the ISOR study. Different results were observed previously, $\varepsilon 2$ allele was associated with obesity, in a study among the population of Croatia's Roma minority ${ }^{22}$. Gene-nutrition interactions can modulate the association between the $\varepsilon 2$ allele and obesity. Indeed, changes in eating habits during the last decade would be responsible for increasing the prevalence of obesity, interacting with the Epsilon alleles ${ }^{23,24}$

The polymorphisms rs439401 and rs4420638 have been associated in some of European population based GWAS-type studies with changes in plasma lipid concentrations $10,15,16$, but few studies have investigated the impact of these polymorphisms on metabolic and cardiovascular traits.

Our results on the Oran population, reported for the first time, that the $\mathrm{T}$ allele of the rs439401 polymorphism increases the risk of arterial hypertension (OR 1.46, 95\% CI [1.12-1.90] $\mathrm{p}=0.006)$. No similar results were reported. In the literature, the T allele of rs439401 is significantly associated with changes in BMI, insulin concentration, waist circumference, and triglyceride concentration. The TT genotype is positively associated with an increase in the values of these parameters only in psychologically stressed individuals ${ }^{25}$. Our results are perhaps indicative of a state of stress of the population, resulting from the changes made in the Algerian population during the last two decades, particularly with the security crisis in the country. These hypotheses require investigations on a larger sample and in which, the stress level must be measured accurately.

The $\mathrm{G}$ allele of rs4420638 seems to confer a protective effect against obesity (OR 0.48, 95\% CI [0.29-0.79] p $=0.004)$, no study had shown interest in measuring association between rs4420638 polymorphism and obesity previously. No association was reported for the rs4420638 with MetS, T2D and HBP similar results were observed in Tunisian population ${ }^{26}$.

The association study of the three genetic variants located in the 19q13.32 region with CHD risk showed no significant association; our results contradict previous studies which have reported that the APOE epsilon polymorphism is a genetic risk factor for $\mathrm{CHD}^{7-10}$. In the same way, the rs439401 and rs4420638 polymor- 
phisms seem to have no effect on the risk of CHD, despite their reported effects on obesity, hypertension and changes in lipid status. Previous studies reported the association of these two variants with CHD. Indeed, several meta-analyses suggest association of the rs4420638 with CHD risk in Americans, Europeans and Asians ${ }^{27,28}$.

The difference in results between the present study and the previous studies describing associations between these polymorphisms studied and the occurrence of CHD could be explained by several factors such as ethnicity, selection criteria of patients and controls, and genetic heterogeneity in the pathogenesis of CHD. In addition, one of the limitations of our study is the relatively small sample size that influences the statistical power. In fact, the sample size is closely tied to statistical power: A larger sample size yields higher power.

The fact that rs4420638 has low linkage disequilibrium with the epsilon polymorphism in our population gives it an advantage over European populations, where these two polymorphisms are in strong linkage disequilibrium $^{1}$. Thus, the study of the impact of rs4420638 would be independent of the effect of epsilon polymorphism, which makes our population very interesting from a genetic point of view for association analysis involving rs4420638 polymorphism.

\section{Conclusion}

Although the studied genetic variants were not associated with the risk of CHD, the $19 \mathrm{q} 13.32$ locus was associated with some of the cardiometabolic disorders in Algerian subjects. The relation of rs439401 alleles with HBP seems perhaps indicative of a state of stress of the population. These hypotheses require, in the future, investigations on a larger sample and in which, the stress level must be measured accurately.

The interaction of gene-nutrition must be investigated, in the future. Indeed, Algerian population has shown many changes in eating habits during the last decade, which might be responsible for increasing prevalence of obesity in our population and which can influence the effect of the three polymorphisms on the studied parameters.

\section{Conclusion}

our findings suggest that these polymorphisms are not involved in genetic susceptibility to CHD in our population; the lack of association between the genetic variants located in the 19q13.32 region and CHD can be explained by the relatively small sample size that influences the statistical power.
Considering their association with obesity and high blood pressure, the studied polymorphisms can be used, in clinical practice, to identify the risk subjects; who once identified, a prevention strategies can be recommended for individual and the public use. However, such studies should be performed on much larger populations in order to deal with the statistical limitations inherent in multiple testing.

\section{Abbreviations \\ ANDRS: Agence Nationale De Recherche en Santé; APOE: Apolipoprotein E; ATRSS: Agence Théma- tique de Recherche en Science de la Santé; BMI: Body mass index; DBP: Diastolic blood pressure; DNA: De- oxyribonucleic acid; d.f.: Degree of freedom; HDL: High-density lipoprotein; ISOR: InSulino-résistance à ORan; LDL: Low-density lipoprotein; MetS: Metabolic Syndrome; SBP: Systolic blood pressure; SNP: Single nucleotide polymorphism; T2D: Type 2 Diabetes,}

\section{Competing interests}

We declare no competing interests.

\section{Acknowledgements}

The ISOR project was funded through a collaboration agreement between (DPGRF) the Direction de la Post-Graduation et de la Recherche-Formation (Algeria) and (INSERM) the Institut National de la Santé et de la Recherche Médicale (France). The work in France was also part-funded by INSERM. The work in Algeria was also part-funded by the Algerian National Agency for the Development of Health Research (ANDRS) and a grant from the Projets Nationaux de Recherche (PNR) program run by the Algerian Direction Générale de la Recherche Scientifique et du Développement Technologique/ Ministère de l'Enseignement Supérieur et de la Recherche Scientifique (DGRSDT/MESRS). We are grateful to Dr Louisa Goumidi, Dr Aline Meirhaeghe and Pr Philippe Amouyel for technical support in DNA extraction, genotyping and statistical analysis, which were performed in their lab (INSERM, U744; Institut Pasteur de Lille, Université Lille Nord de France, Lille, France)

\section{References}

1. Boulenouar H, Benchekor SM, Meroufel DN, Hetraf SAL, Djellouli HO, Hermant X, et al. Impact of APOE gene polymorphisms on the lipid profile in an Algerian population. Lipids Health Dis. 2013;12.

2. Murray CJ, Lopez AD. Mortality by cause for eight 
regions of the world: Global Burden of Disease Study. Lancet. 1997;349(9061):1269-76.

3. Watkins H, Farrall M. Genetic susceptibility to coronary artery disease: from promise to progress. Nature Reviews Genetics. 2006;7(3):163-73.

4. Ginsberg HN, Kris-Etherton P, Dennis B, Elmer PJ, Ershow A, Lefevre M, et al. Effects of reducing dietary saturated fatty acids on plasma lipids and lipoproteins in healthy subjects: the DELTA Study, protocol 1. Arteriosclerosis, Thrombosis, and Vascular Biology. 1998;18(3):4419.

5. Savolainen MJ, Kesaniemi YA. Effects of alcohol on lipoproteins in relation to coronary heart disease. Current Opinion in Lipidology. 1995;6(4):243-50.

6. Gielen S, Schuler G, Hambrecht R. Exercise training in coronary artery disease and coronary vasomotion. Circulation. 2001;103(1):E1-6.

7. Wilson PW, Schaefer EJ, Larson MG, Ordovas JM. Apolipoprotein $\mathrm{E}$ alleles and risk of coronary disease. A meta-analysis. Arteriosclerosis, Thrombosis, and Vascular Biology. 1996;16(10):1250-5.

8. Song Y, Stampfer MJ, Liu S. Meta-analysis: apolipoprotein $\mathrm{E}$ genotypes and risk for coronary heart disease. Annals of Internal Medicine. 2004;141(2):137-47.

9. Bennet AM, Di Angelantonio E, Ye Z, Wensley F, Dahlin A, Ahlbom A, et al. Association of apolipoprotein $\mathrm{E}$ genotypes with lipid levels and coronary risk. JAMA. 2007;298(11):1300-11.

10. Teslovich TM, Musunuru K, Smith AV, Edmondson AC, Stylianou IM, Koseki M, et al. Biological, clinical and population relevance of 95 loci for blood lipids. Nature. 2010;466(7307):707-13.

11. Dallongeville J, Lussier-Cacan S, Davignon J. Modulation of plasma triglyceride levels by APOE phenotype: a meta-analysis. Journal of Lipid Research. 1992;33(4):44754.

12. Wilson PW, Myers RH, Larson MG, Ordovas JM, Wolf PA, Schaefer EJ. Apolipoprotein E alleles, dyslipidemia, and coronary heart disease. The Framingham Offspring Study. JAMA. 1994;272(21):1666-71.

13. Costanza MC, Beer-Borst S, James RW, Gaspoz JM, Morabia A. Consistency between cross-sectional and longitudinal SNP: blood lipid associations. European Journal of Epidemiology. 2012;27(2):131-8.

14. Petkeviciene J, Smalinskiene A, Luksiene DI, Jureniene K, Ramazauskiene V, Klumbiene J, et al. Associations between apolipoprotein $\mathrm{E}$ genotype, diet, body mass index, and serum lipids in Lithuanian adult population. PloS One. 2012;7(7):e41525.

15. Aulchenko YS, Ripatti S, Lindqvist I, Boomsma D, Heid IM, Pramstaller PP, et al. Loci influencing lipid levels and coronary heart disease risk in 16 European population cohorts. Nature Genetics. 2009;41(1):47-55.

16. Kathiresan S, Melander O, Guiducci C, Surti A, Burtt NP, Rieder MJ, et al. Six new loci associated with blood low-density lipoprotein cholesterol, high-density lipoprotein cholesterol or triglycerides in humans. $\mathrm{Na}$ ture Genetics. 2008;40(2):189-97.

17. Willer CJ, Speliotes EK, Loos RJ, Li S, Lindgren CM, Heid IM, et al. Six new loci associated with body mass index highlight a neuronal influence on body weight regulation. Nature Genetics. 2009;41(1):25-34.

18. Liu Y, Zhou D, Zhang Z, Song Y, Zhang D, Zhao T, et al. Effects of genetic variants on lipid parameters and dyslipidemia in a Chinese population. Journal of Lipid Research. 2011;52(2):354-60.

19. Alberti KG, Zimmet P, Shaw J. The metabolic syndrome--a new worldwide definition. Lancet. 2005;366(9491):1059-62.

20. Gavin JR, 3rd. New classification and diagnostic criteria for diabetes mellitus. Clinical cornerstone. 1998;1(3):112.

21. Chalmers J, MacMahon S, Mancia G, Whitworth J, Beilin L, Hansson L, et al. 1999 World Health Organization-International Society of Hypertension Guidelines for the management of hypertension. Guidelines sub-committee of the World Health Organization. Clin Exp Hypertens. 1999;21(5-6):1009-60.

22. Zeljko HM, Skaric-Juric T, Narancic NS, Tomas Z, Baresic A, Salihovic MP, et al. E2 allele of the apolipoprotein $\mathrm{E}$ gene polymorphism is predictive for obesity status in Roma minority population of Croatia. Lipids Health Dis. 2011;10:9.

23. Boer JM, Ehnholm C, Menzel HJ, Havekes LM, Rosseneu M, O'Reilly DS, et al. Interactions between lifestyle-related factors and the APOE polymorphism on plasma lipids and apolipoproteins. The EARS Study. European Atherosclerosis Research Study. Arteriosclerosis, Thrombosis, and Vascular Biology. 1997;17(9):1675-81.

24. Talmud PJ. Gene-environment interaction and its impact on coronary heart disease risk. Nutrition, metabolism, and cardiovascular diseases: NMCD. 2007;17(2):14852.

25. Kring SI, Brummett BH, Barefoot J, Garrett ME, Ashley-Koch AE, Boyle SH, et al. Impact of psychological stress on the associations between apolipoprotein $\mathrm{E}$ variants and metabolic traits: findings in an American sample of caregivers and controls. Psychosomatic Medicine. 2010;72(5):427-33.

26. Elouej S, Rejeb I, Attaoua R, Nagara M, Sallem OK, Kamoun I, et al. Gender-specific associations of genetic variants with metabolic syndrome components in the 
Tunisian population. Endocrine Research. 2016;41(4):3009.

27. Huang Y, Ye HD, Gao X, Nie S, Hong QX, Ji HH, et al. Significant interaction of APOE rs4420638 polymorphism with HDL-C and APOA-I levels in coronary heart disease in Han Chinese men. Genetics and Molecular Research : GMR. 2015;14(4):13414-24.
28. Grallert H, Dupuis J, Bis JC, Dehghan A, Barbalic M, Baumert J, et al. Eight genetic loci associated with variation in lipoprotein-associated phospholipase A2 mass and activity and coronary heart disease: meta-analysis of genome-wide association studies from five community-based studies. European heart journal. 2012;33(2):238-51 